\title{
The mind behind me
}

\section{How and why does the brain generate a sense of self?}

\section{The Face in the Mirror: The Search for the Origins of Consciousness}

by Julian Keenan with Gordon G. Gallup Jr \& Dean Falk

Harper Collins: 2003. 304 pp.

US\$24.95, Can\$38.95

\section{Marc Hauser}

In the film Memento, the main character, Leonard Shelby, suffers an injury to the head that creates a nightmarish problem. Although his experiences before the injury are clear, everything that has happened after it is unfamiliar, even events that occurred just moments before. Because the mind's recording device is broken, things are experienced but never recalled. Shelby's autobiography is frozen in the past.

It is hard for us to imagine what it would be like to lack this kind of memory. It would certainly rob us of one component of our sense of self, of what it was like to have experienced different things in the world. We would not be able to judge whether or not we like something; we would have to resample each event on the moment. Even if we resorted to writing down each experience, as Shelby tried to do, we would be constrained by the relatively impoverished connection between subjective experience and language.

Understanding how this sense of self is constructed and represented in the brain is the focus of The Face in the Mirror, a book written by three scientists who have all made significant contributions to their respective disciplines. Cognitive neuroscientist Julian Keenan is known for his neuroimaging studies of self-recognition; the comparative psychologist Gordon Gallup invented the mirror test, which has been used to explore selfrecognition in animals and human infants; and the biological anthropologist Dean Falk has documented several important historical patterns of human brain evolution. Although they are all co-authors of the book, it is written in Keenan's first-person voice.

According to Keenan, the goal of the book is "to tell the story of self-awareness, why it exists, where it came from, and where in the brain we might locate this astonishing ability. The findings and conclusions we have reached are quite amazing and contrary to what many neuroscientists believe." This is a highly ambitious research programme. How well does he fare?

The book begins by laying the relevant groundwork, including the history of some of the ideas that drive research on the sense of self, in terms of both self-recognition and self-awareness. Although Keenan stays clear

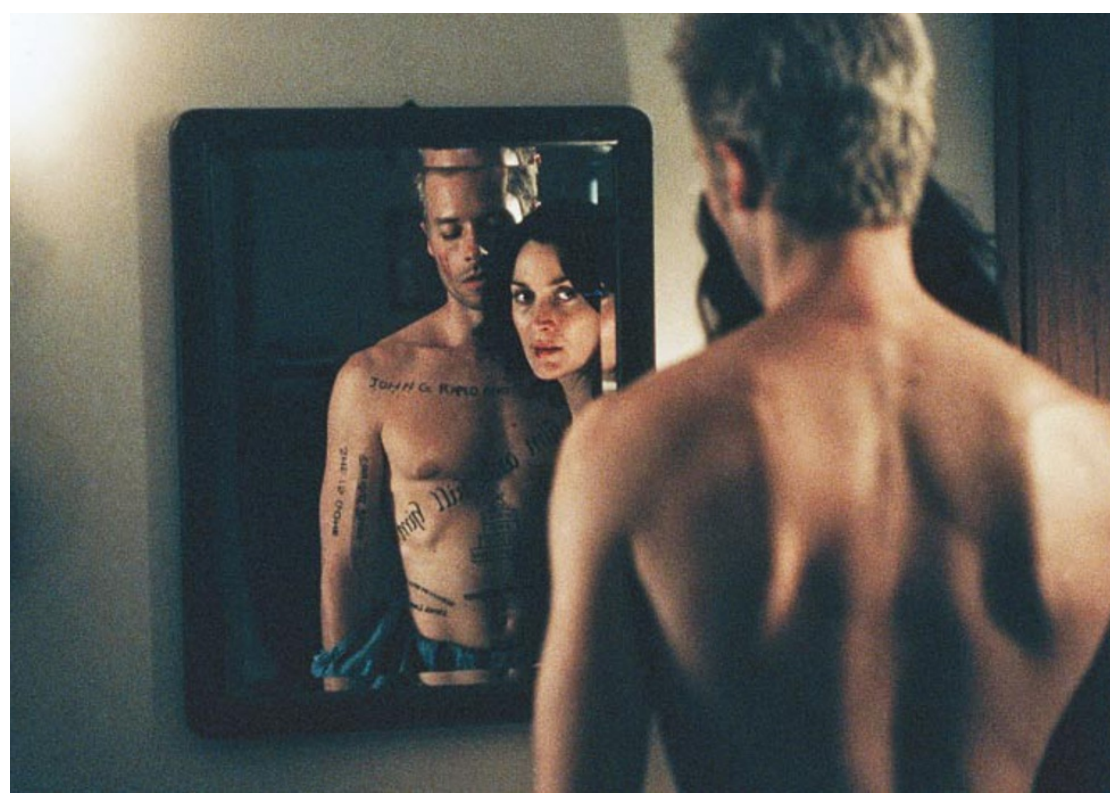

A familiar face: our sense of self reflects our capacity for self-recognition and self-awareness.

of many of the interesting philosophical issues concerning subjective experience, and offers no insights into the selective pressures on or adaptive significance of our sense of self, the issues that he raises are nonetheless fascinating.

For example, when an individual recognizes herself in the mirror, what does this tell us, if anything, about what she is thinking? The answer to this question is important because it has implications for understanding the nature of animal and human minds. If, as Gallup proposed more than 20 years ago, mirror self-recognition provides a litmus test for self-awareness, then we can use it to explore which animals, and which humans, have this kind of a conscious mental life. If not, then other tests are needed to assess whether non-linguistic or pre-linguistic organisms have the capacity for self-awareness. Answering this question should also help us to refine our interpretation of neurophysiological and neuropsychological data from both humans and other animals.

Here, in a nutshell, is the reported evidence. First, not all animals show mirror self-recognition. This capacity seems to be restricted to the great apes and humans over the age of 18-24 months. Keenan claims that animals with self-recognition also have the capacity to attribute mental states to othersthey have a theory of mind. More specifically, at about the time that children acquire mirror self-recognition, they also acquire a rudimentary sense of self-awareness. Consequently, so Keenan argues, mirror self-recognition provides a litmus test for a theory of mind.

Second, patients with a loss of self-awareness often have deficits in mirror recognition. In some of these patients, especially those with anosognosia, which involves paralysis to one side of the body accompanied by complete denial that the deficit exists, the damage is typically in the right hemisphere. Third, neuroimaging and psychophysical studies indicate that the right hemisphere has a dominant role in selfrecognition. So Keenan suggests that the capacity for self-recognition is deeply connected to the capacity for self-awareness; that this connection evolved before the emergence of our species; and that our sense of self resides in the right hemisphere.

This is an intriguing hypothesis, with some supporting evidence, but there are three problems. To begin with, there are some situations in which humans fail to show mirror self-recognition, but still maintain a rich sense of who they are and what they believe and feel. Some patients with prosopagnosia - a selective face-recognition deficit - fail to recognize their own mirror image or a picture of their own face, and this realization strikes at the core of their sense of self. So there is not a necessary relationship between self-recognition and self-awareness. Similarly, the evidence that Keenan cites supporting a relationship between the mirror test and a theory of mind in apes has been dismissed as inconclusive by others, including Daniel Povinelli, who carried out the original research on the idea. Oddly, Keenan does not mention 
the much stronger evidence that has been compiled in the past three years by Brian Hare, Michael Tomasello and Josep Call, but even this work says nothing about the content of a chimpanzee's beliefs.

A second problem is that research on the developing theory of mind goes well beyond the literature reviewed by Keenan, and raises significant problems for his theory. Specifically, it is now clear that for many cognitive capacities, including the theory of mind, children have implicit knowledge long before it is explicit; using an explicit measure such as the mirror test might cause us to miss an earlier capacity. Furthermore, part of the challenge for the child in acquiring an explicit theory of mind is that it requires considerable executive control, something that young children lack because of the relative immaturity of the frontal lobes. This means that a child's inability to understand what others believe is not necessarily a reflection of the challenges it faces in attributing similar or different beliefs to others; rather, the difficulty may stem from the challenge associated with inhibiting personal beliefs in order to make accurate judgements about others.

Third, even if our sense of self is located in the right hemisphere - and there is chauvinism against this side of the brain from neuroscientists who think that the left hemisphere does all the heavy intellectual lifting — this does not help our understanding of how the brain generates a feeling of personal experience, of guilt, awe, shame or despair. It wouldn't help us to understand why Shelby

\section{New in paperback}

Science, Money, and Politics: Political Triumph and Ethical Erosion

by Daniel S. Greenberg

University of Chicago Press, \$20, £14

"...the value of this book [is] as a unique and revealing perspective on the way that the science-funding process actually works in Washington. The picture it paints is not a flattering one. But - unlike many of those he writes about - Greenberg is not out to make friends in high places." David Dickson, Nature 413, 355-356 (2001).

\section{The Future of Life}

by Edward O. Wilson

Abacus, £8.99

"[Wilson] accurately and passionately tells the story of the disappearance of many of the only living beings we know of in the Universe key components of humanity's natural capital." Paul R. Ehrlich, Nature 417, 21-22 (2002).

\section{Secret Agents: The Menace of Emerging} Infections

by Madeline Drexler

Penguin, $\$ 15.00$ is distraught, or whether medical technology might someday reverse these cases of brain damage.

In the end, however, Keenan and his coauthors have assembled a rich set of evidence that will contribute to what is certainly one of the most interesting topics in the sciences of the mind: our sense of self. At least, that's what I think, I think.

Marc Hauser is at the Primate Cognitive

Neuroscience Laboratory and the Department of Psychology, Harvard University, Cambridge, Massachusetts 02138, USA.

\section{A flexible theory of evolution}

\section{Developmental Plasticity and \\ Evolution}

by Mary Jane West-Eberhard

Oxford University Press: 2003.720 pp. \$100,

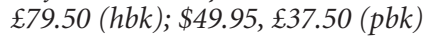

\section{Gerdien de Jong and Ross H. Crozier}

It is an ancient truism that what evolves is the developmental system, from which it follows that genetics, development and evolution are interwoven. Genetics and evolution were integrated long ago in the synthetic theory of evolution; development has only lately rejoined the evolutionary fold. The field of evolutionary developmental biology thus created is mostly concerned with patterns of evolution, comparing the genetic basis of evolutionary changes in development, rather than with the dynamics of evolutionary changes. Its sister, developmental evolutionary biology, studies how selection works on development to produce the adaptive phenotype, necessarily becoming linked to evolutionary ecology. This is a field worth pursuing, and Mary Jane West-Eberhard's Developmental Plasticity and Evolution certainly belongs within it, but the question is whether the book does the field good service.

For West-Eberhard the phenotype is central. But this is not the bleak 'genotype + environment $=$ phenotype' taught to firstyear students, but rather a vibrant, living, changing phenotypic whole, far from dreary genetic determinism. The phenotype is developmentally plastic, changing in many ways in response to many environmental challenges. To be alive is to be developmentally plastic. West-Eberhard envisages a synthetic theory of evolution and development in which environmentally induced phenotypic change gives rise to adaptive evolution as readily as, or even more readily than, mutationally induced phenotypic change.

The main evolutionary process, in WestEberhard's universe, involves environmental change, phenotypic accommodation and genetic accommodation. An environmental change elicits a developmentally plastic response, and phenotypic accommodation - the immediate adjustment to a change resulting from the multidimensional adaptive flexibility of the phenotype - ameliorates its harm to individuals. New phenotypes resulting from this developmental plasticity are selected. A change in allele frequency genetic accommodation - improves and incorporates the change. In this way the environment becomes a crucial participant in the generation and selection of adaptive design.

In West-Eberhard's view, this sequence of developmental plasticity, phenotypic accommodation and genetic accommodation is the mechanism responsible for (nearly) all

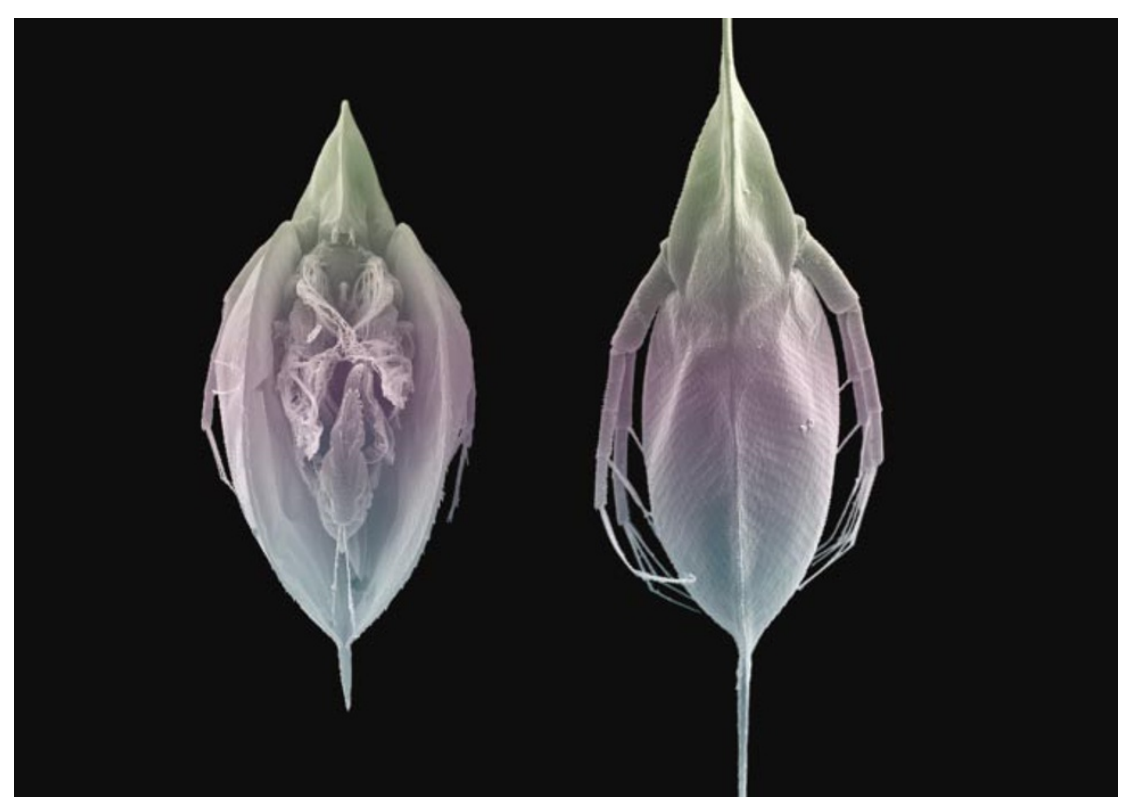

Spot the difference: the water flea Daphnia readily changes shape in response to its environment. 